\title{
Efecto del Cigarrillo Electrónico sobre los Espermatozoides Humanos: Aproximación in vitro
}

\section{Effect of Electronic Cigarette on Human Sperm: in vitro Approximation}

\author{
Mateo Morales Velásquez ${ }^{1} \quad$ Jenniffer Puerta Suarez ${ }^{1 \odot} \quad$ Walter D. Cardona Maya ${ }^{1 \odot}$ \\ ${ }^{1}$ Grupo de Reproducción, Departamento de Microbiología y \\ Parasitología, Facultad de Medicina, Universidad de Antioquia, \\ Medellín, Colombia \\ Address for correspondence Walter D. Cardona Maya, Bact, MSc, \\ PhD, Departamento de Microbiología y Parasitología, Facultad de \\ Medicina, Universidad de Antioquia, Carrera 53 \# 61-30, Laboratorio \\ Urol Colomb 2020;29:7-13. \\ 534, Medellín, Colombia (e-mail: wdario.cardona@udea.edu.co).
}

\section{Resumen \\ Palabras clave \\ - cigarrillo electrónico \\ - cigarrillo \\ - espermatozoides \\ - infertilidad \\ - calidad seminal}

Introducción Los cigarrillos electrónicos (E-Cig) aparecieron en el mercado hace 15 años como una alternativa para combatir el consumo de tabaco, un problema de salud pública, sin embargo, su efecto sobre la salud reproductiva no ha sido completamente evaluado. El objetivo del presente estudio, fue evaluar los efectos de los E-Cig sobre los espermatozoides humanos in vitro.

Métodos Un dispositivo para incubar en contacto directo a los espermatozoides con el vapor a partir de dos esencias diferentes (TNT y Sugar Drizzle), el humo del cigarrillo o aire como control negativo fue construido. Adicionalmente, usando el mismo dispositivo, se elaboró el extracto a partir del aire, de las dos esencias con el E-Cig y con el cigarrillo Piel Roja sin filtro en medio para incubar en contacto indirecto con los espermatozoides.

Resultados Se observó disminución de la movilidad con el E-Cig en más del 38\% en el tiempo y una disminución de la viabilidad en más del $18 \%$ que se mantenían con el tiempo mientras que con el cigarrillo convencional afectaba la movilidad y la viabilidad en casi el $100 \%$. Finalmente se encontró que se afecta más la movilidad de los espermatozoides con la esencia de Sugar Drizzle que contaba con menos concentración de nicotina que con la esencia de TNT.

Conclusiones En conclusión, tanto el cigarrillo convencional como el E-Cig afectan los parámetros seminales (movilidad y viabilidad), y se postula que el uso del E-Cig, aunque es menos citotóxico y afecta menos que el cigarrillo convencional, también puede generar a largo plazo problemas de infertilidad.

\section{Abstract} alternative to combat tobacco use, a public health problem, however, its effect on

received January 15, 2019 accepted April 17, 2019
DOI https://doi.org/ $10.1055 / \mathrm{s}-0039-1691783$ ISSN 0120-789X. e ISSN 2027-0119.
Copyright ( 2020 , Sociedad Colombiana License terms de Urología. Publicado por Thieme Revinter Publicações Ltda., Rio de Janeiro, Brazil. Todos los derechos reservados.

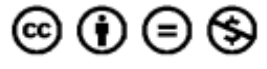




\author{
Keywords \\ - electronic cigarette \\ - cigarette \\ - spermatozoa \\ - infertility \\ - semen quality
}

reproductive health has not been fully evaluated. The objective of the present study was to evaluate the effects of E-Cig on human sperm in vitro.

Methods A device to incubate sperm in direct contact with steam from two different scents (TNT and Sugar Drizzle), cigarette smoke or air as a negative control was built. Additionally, using the same device, extracts were elaborated from the air, from the two essences with the E-Cig and with the Red Skin cigarette without a filter in the medium to incubate in indirect contact with sperm cells.

Results Decreased motility with E-Cig was observed in more than $38 \%$ over time and a decrease in viability in more than $18 \%$ that was maintained over time while with the conventional cigarette affected motility and viability in almost $100 \%$. Finally, we found that sperm motility of the sperm is more affected with the essence of Sugar Drizzle that had less nicotine concentration than with TNT essence.

Conclusions In conclusion, both the conventional cigarette and the E-Cig affect the seminal parameters (motility and viability) and it is postulated that the use of the E-Cig, although it is less cytotoxic and affects less than the conventional cigarette can also generate long-term infertility problems.

\section{Introducción}

El consumo de tabaco es la causa más prevenible de mortalidad en el mundo, aunque genera la muerte de más de cinco millones de personas al año. Según la Organización Mundial de la Salud ${ }^{1}$ si las tendencias actuales continúan para el año 2030 el consumo de tabaco ocasionará más de ocho millones de muertes cada año y para finales del siglo se estima que ocasionará más de 1000 millones de muertes. ${ }^{1} \mathrm{El}$ cigarrillo está compuesto por hojas de tabaco (50\%), tabaco reconstituido (30\%) y tabaco expandido con dióxido de carbono $(20 \%)^{2}$ y el humo generado durante su consumo produce más de 7000 componentes, de los cuales alrededor de 70 son cancerígenos: arsénico, benceno, berilio, 1,3butadieno, cadmio, cromo, óxido de etileno, níquel, y cloruro de vinilo, entre otros productos tóxicos. ${ }^{2}$

Fumar cigarrillo ha sido reportado como perjudicial para la función reproductiva masculina. ${ }^{3-5}$ Un meta análisis que incluyó 27 estudios demostró que el consumo de tabaco está asociado con la disminución de la concentración y la movilidad espermática y con alteraciones en la morfología de los espermatozoides, parámetros que se afectan proporcionalmente a la dosis y al tiempo de exposición al cigarrillo. ${ }^{6,7}$ Además, el $39 \%$ de los hombres no fumadores son normozoospérmicos en contraste con un $3 \%$ de los fumadores, en los cuales se ha observado un número más alto de astenozoospermia y astenoteratozoospermia. ${ }^{3}$ Incluso, se plantean asociaciones entre fumar cigarrillo con un incremento en el número de leucocitos seminales y de especies reactivas de oxígeno, condiciones que inducen al estrés oxidativo, generando daño del ADN espermático lo cual podría inducir anormalidades cromosómicas. ${ }^{8-10}$

Con el fin de aminorar los efectos ligados al uso del cigarrillo convencional, desde hace algunos años aparecieron los cigarrillos electrónicos (E-Cig), también conocidos como vaporizadores o sistemas de suministro de nicotina, los cuales fueron inventados por el farmacéutico chino Hon Lik. ${ }^{11}$ En la actualidad, los E-Cig son aceptados mundialmente y muchos países están legalizando su uso y la venta de esencias con nicotina, con el propósito de usar los E-Cig como una alternativa para que los fumadores abandonen el tabaco ${ }^{12}$ y así lograr reducir los índices de mortalidad asociados al consumo de cigarrillo. ${ }^{13}$ Se ha reportado que el uso de los E-Cig tiene un fuerte impacto en ayudar a las personas a abandonar el consumo de cigarrillo, pero especialmente evitando los efectos negativos colaterales que se generan al dejar la nicotina de manera súbita. ${ }^{14}$

Específicamente, los E-Cig utilizan una batería y una resistencia con el fin de administrar la nicotina inhalada mediante la vaporización de una solución compuesta por propilenglicol, glicerol y esencias concentradas tanto naturales como artificiales conocidas como E-Liquids ${ }^{15}$ con sabores a canela, melón, piña y banana, e incluso tabaco, entre otros, los cuales son aprobados y ampliamente utilizados en productos de consumo humano. ${ }^{16}$ Una de las ventajas del E-Cig es que al hacer uso de este (vaporear), se deja a un lado la combustión directa generada durante el consumo del cigarrillo convencional ${ }^{17}$ por lo que los ELiquids y aerosoles generados a partir de los E-Cig contienen menos químicos e inducen menos citotoxicidad y efectos adversos que el cigarrillo convencional. ${ }^{18-21}$

Según Rezk y col., ${ }^{13}$ para el año 2014, existían aproximadamente 500 marcas de E-Cig y alrededor de 8000 esencias con concentraciones variables de nicotina. El Centro de Control y Prevención de Enfermedades (CDC) reporta que, debido a su fácil acceso, los E-Cig están presentando un crecimiento exponencial entre jóvenes y adolescentes en los cuales su uso se incrementó en un 
$800 \%$, incluso permitiendo una reducción en el consumo de cigarrillo convencional en esa población en más de un $6 \%{ }^{2}$

Existen pocas referencias sobre las consecuencias del uso del cigarrillo electrónico en el organismo y menos aún sobre el tracto reproductivo. Basados en lo anterior, el objetivo del presente estudio fue evaluar los efectos de los E-Cig sobre los espermatozoides humanos in vitro.

\section{Métodos}

\section{Muestras Seminales}

Se incluyeron 9 muestras de semen de individuos sanos con parámetros seminales normales (normozoospérmicos), colectadas mediante masturbación después de un periodo de abstinencia sexual de 3 a 7 días. A cada muestra se le cuantificó la movilidad y la viabilidad espermática mediante microscopía siguiendo los lineamientos establecidos en el manual de análisis seminal de la OMS. ${ }^{22}$

\section{Incubación Directa Entre Espermatozoides y Producto de los E-Cig}

En un tubo cónico de $50 \mathrm{~mL}$ con la tapa modificada con el fin de incluir una manguera para entrada y una para salida de vapor, humo o aire por medio de una jeringa (-Figura 1), se incubaron 500uL de la muestra seminal con 500uL de tampón fosfato salino (PBS, SIGMA®, Steinheim, Alemania) y se procedió a circular por la manguera de entrada el aire (control negativo), el vapor producido por el E-Cig (Smok Stick V8 Baby, Guangdong, China) con cada una de las dos esencias: tabaco con 6 mg de nicotina The Next Tobacco (TNT) (Innevape, California, EE. UU.) o esencia dulce de $3 \mathrm{mg}$ de nicotina Sugar Drizzle (Cuttwood, California, EE. UU.) o el humo del cigarrillo sin filtro (Piel Roja, Medellín, Colombia, adquirido por Philip Morris, Nueva York, EE.UU.), con el propósito de hacerlos entrar en contacto con los espermatozoides a través de una punta de $1000 \mathrm{uL}$ (- Figura 1).

El aire se pasó durante 2 segundos cada 45 segundos durante 11 minutos (15 veces), el vapor se indujo activando el vaporizador y generando el vacío con la jeringa de $50 \mathrm{~mL}$ durante 4 segundos alcanzando el tope de la jeringa, y cada 45 segundos hasta completar 12 minutos (15 veces), mientras que para el cigarrillo se generó el vacío con la jeringa durante 2 segundos hasta el tope de la jeringa, y cada 45 segundos hasta que se consumiera todo el cigarrillo (-Figura 1). Cada muestra se procesó al tiempo con aire, vapor o cigarrillo, evaluando la movilidad y la viabilidad espermática al tiempo cero, 30 y 60 minutos.

\section{Temperatura Durante Incubación Directa}

Con el propósito de determinar la temperatura durante el ensayo, usando una cámara térmica (Fluke Ti100, Everett, Washington, EE.UU.), se realizó el seguimiento del comportamiento de la temperatura durante los ensayos (datos no mostrados) y no se encontraron variaciones en la temperatura de la muestra seminal, aunque sí un incremento hasta los $71,4^{\circ} \mathrm{C}$ en la temperatura del E-Cig como era de esperar.

\section{Preparación Extractos de E-Cig y Cigarrillo Convencional}

Para el desarrollo de este trabajo, los extractos fueron producidos en medio HAM F10 (Thermo Fisher Scientific, Waltham, Massachusetts, EE.UU.), para realizar las incubaciones con los espermatozoides humanos.

Los extractos de vapor de cada una de las esencias (TNT o Sugar Drizzle), o del cigarrillo Piel Roja sin filtro o del aire, se produjeron simulando el uso del E-Cig o el cigarrillo. Brevemente, generando el vacío por medio de la jeringa, se pasó el vapor por el medio HAM F10 (Thermo Fisher Scientific, Waltham, Massachusetts, EE.UU.) suplementado con 10\% de Suero Bovino Fetal (SBF, GIBCO®, Thermo Fisher Scientific, Waltham, Massachusetts EE.UU.), $10 \%$ de penicilinaestreptomicina (GIBCO®, Thermo Fisher Scientific, Waltham, Massachusetts, EE.UU.) y $1 \%$ de gentamicina (GENFAR®, Colombia), durante 4 segundos cada 60 segundos por un total de 45 minutos (45 veces) para el E-Cig, durante 2 segundos cada 30 segundos hasta que se consumieran tres
A.

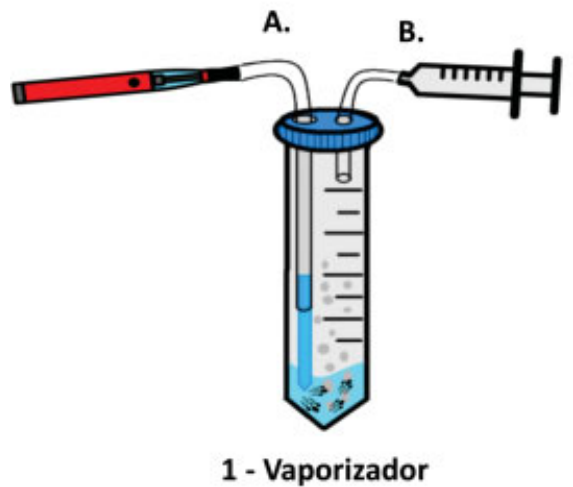

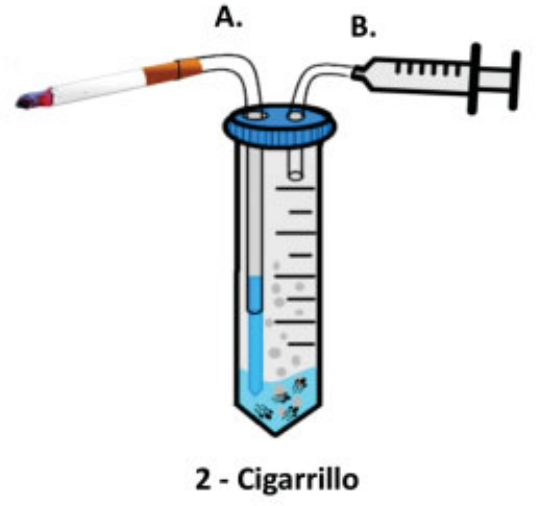

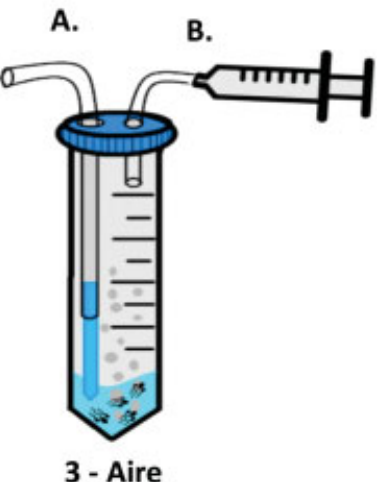

Fig. 1 Dispositivo para la absorción y el flujo de vapor, aire o cigarrillo. A cada tubo de $50 \mathrm{~mL}$ se le adaptaron dos mangueras de polipropileno, una donde se adaptó por un lado una punta de 1000 uL que estuvo en contacto con la muestra y por el otro lado adaptada al vaporizador ( $1 \mathrm{~A}$ ), el cigarrillo (2A) o libre para permitir el flujo del aire (3A). En el otro extremo a la otra manguera (B), se le adaptó una jeringa de $50 \mathrm{~mL}$ la cual se utilizó para generar el vacío y permitir la absorción y el flujo del vapor, del humo o del aire. 
cigarrillos para el extracto de cigarrillo, o durante 2 segundos cada 30 segundos (45 veces) para el extracto control con aire solo. Los resultados de esas soluciones fueron considerados como extractos al $100 \%$.

\section{Incubación Indirecta Entre Espermatozoides y Productos del E-Cig y el Cigarrillo Convencional}

Las muestras seminales normozoospérmicas después de realizada la valoración microscópica inicial fueron incubadas con cada extracto generado en medio HAM F10 (TNT, Sugar Drizzle, cigarrillo Piel Roja o aire), en dos concentraciones diferentes (100\% y 50\%) determinando tanto la movilidad como la viabilidad a los 0 y 60 minutos.

\section{Análisis Estadístico}

Todos los valores fueron expresados como media y desviación estándar. Las comparaciones fueron realizadas usando análisis de la varianza (ANOVA) mediante el programa estadístico GraphPad Prism 7.0 (La Jolla, California, EE.UU.) y las diferencias fueron consideradas estadísticamente significativas si el valor de $p<0,05$.

\section{Resultados}

\section{Procesamiento Directo}

El vapor y los componentes generados a partir del uso del vaporizador mostraron una disminución de los parámetros espermáticos (movilidad y vialidad), con ambas esencias (-Figura 2A-B y 3A-B) respecto a la muestra tratada con aire, en la cual la movilidad disminuyó al tiempo cero en $55 \%$ para el caso de TNT y en $71 \%$ durante el procesamiento con la esencia de Sugar Drizzle, valores que se mantuvieron en el tiempo. Mientras que el humo y los componentes generados a partir del cigarrillo mostraron afectar totalmente la movilidad desde el tiempo cero (-Figura 2A y $3 \mathbf{3 A}$ ) e inducir una disminución de la viabilidad de los espermatozoides (entre $96 \%$ y 100\%) respecto al control negativo con el aire al mismo tiempo (-Figura 2B у 3B).

\section{Incubación Indirecta con los Extractos}

En los extractos de ambas esencias se observa al igual que en el procesamiento directo una disminución de la movilidad en $38 \%$ con TNT y en $45 \%$ con Sugar Drizzle en contraste con el
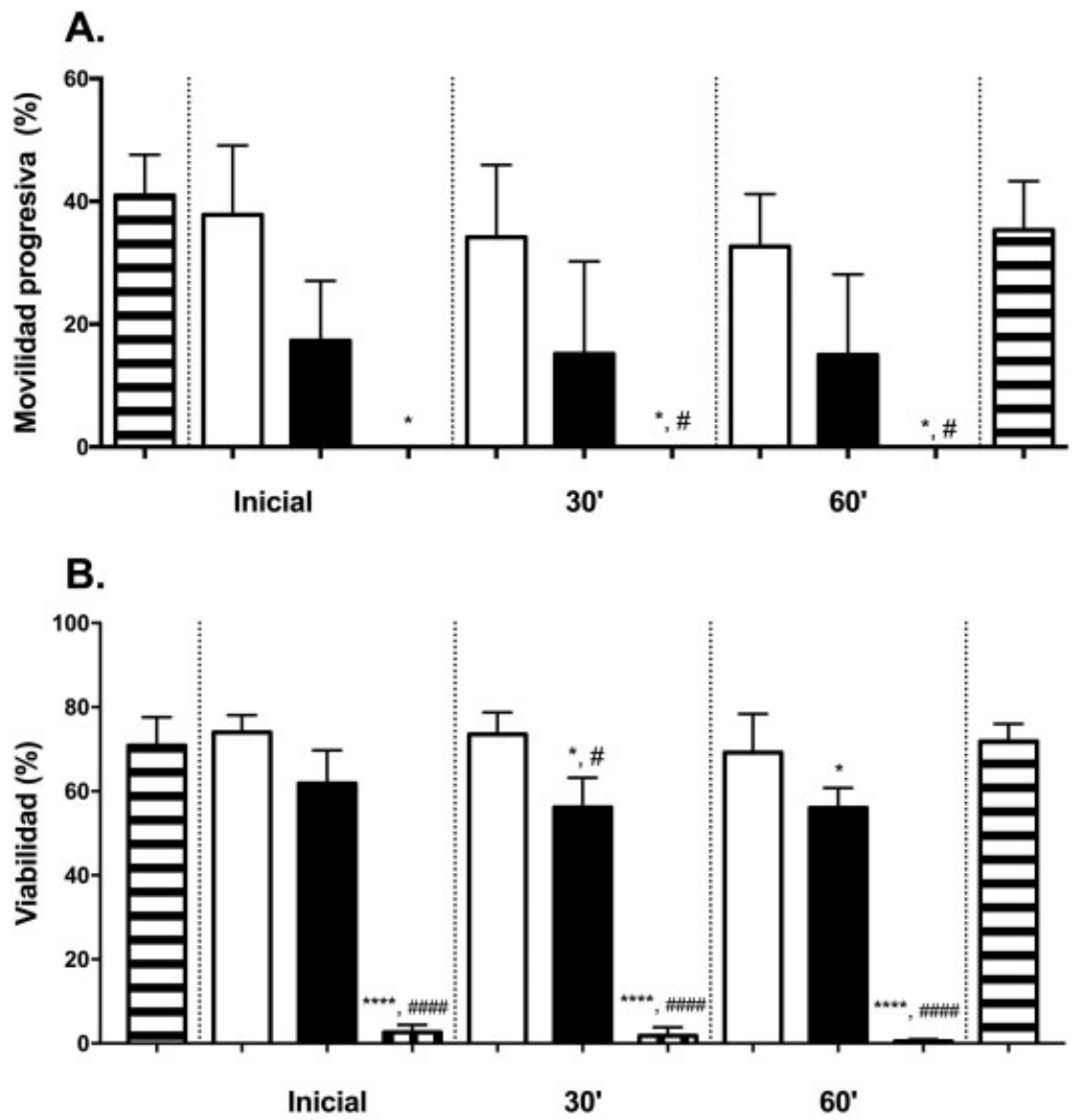

\section{Tiempo}

Fig. 2 Efectos del cigarrillo electrónico con esencia de tabaco (TNT) y del cigarrillo convencional sobre (A) la movilidad (\%) y (B) la viabilidad espermática (\%) con respecto al tiempo. ${ }^{*}$ Comparación con respecto a la muestra inicial. ${ }^{\#}$ Comparación con respecto a la muestra de aire del

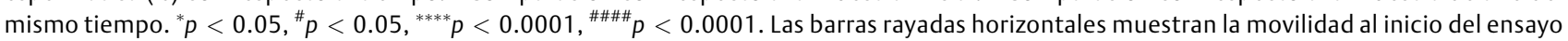
$\mathrm{y}$ al final del ensayo. 


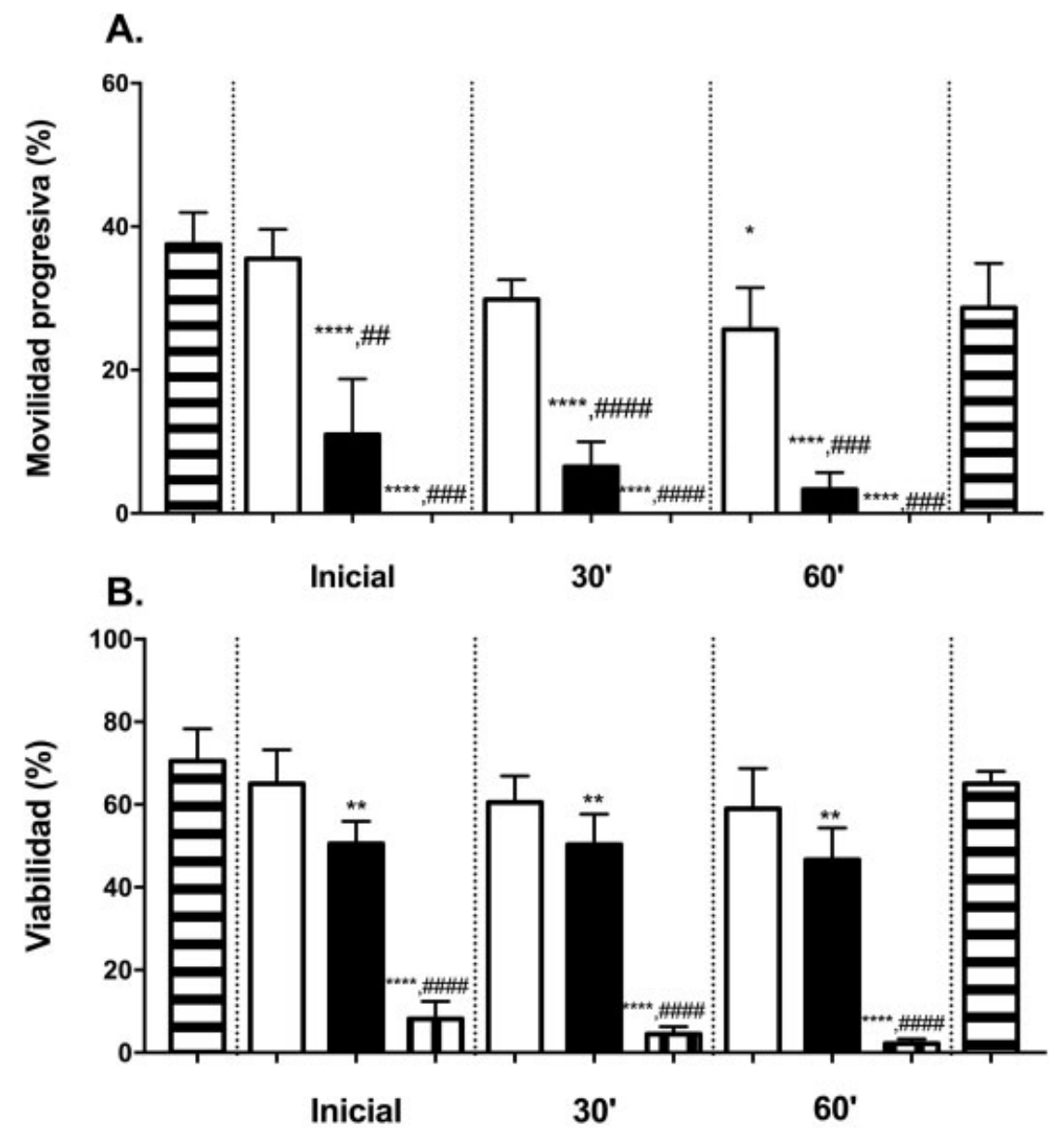

\section{Tiempo}

Fig. 3 Efectos del cigarrillo electrónico con esencia dulce (Sugar Drizzle) y del cigarrillo convencional sobre (A) la movilidad (\%) y (B) la viabilidad espermática (\%) con respecto al tiempo. ${ }^{*}$ Comparación con respecto a la muestra inicial. ${ }^{\#}$ Comparación con respecto a la muestra de aire del

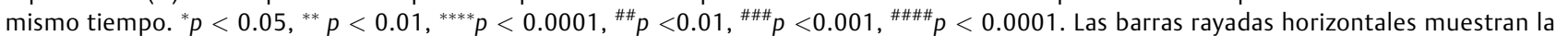
movilidad al inicio del ensayo y al final del ensayo.

extracto de cigarrillo en el cual disminuye al $100 \%$ de la movilidad ( - Figura 4A). De manera similar, la viabilidad espermática se afectó con los extractos de las esencias, aunque el extracto de cigarrillo genera una disminución más pronunciada (- Figura 4B).

\section{Discusión}

En el presente estudio, se realizó la evaluación in vitro del efecto de los componentes generados a partir del uso del ECig y el cigarrillo convencional sobre los parámetros seminales humanos.

En conjunto, los hallazgos de este estudio evidencian que la movilidad inicial de las muestras seminales se afecta por el vaporizador independiente de la concentración de nicotina $(6 \mathrm{mg} / \mathrm{mL}$ o $3 \mathrm{mg} / \mathrm{mL})$, paradójicamente a menor concentración de nicotina los efectos sobre los espermatozoides son más marcados, resultados congruentes con los reportados previamente por El Golli y col., ${ }^{23}$ los cuales fueron los primeros en reportar que las esencias sin nicotina de los cigarrillos electrónicos aplicadas directamente en testículos de roedores, afectan la viabilidad espermática. La nicotina es conocida además por inhibir la biosíntesis de testosterona en las células de Leydig en ratones la cual juega un papel importante en la espermatogénesis por lo que reduce la producción y maduración espermática. ${ }^{24}$

Algunos estudios en roedores reportan que la nicotina afecta los parámetros espermáticos, principalmente la concentración, la movilidad y la viabilidad. ${ }^{24,25}$ La nicotina incrementa los radicales libres de oxígeno por interrumpir la cadena respiratoria mitocondrial e induce un incremento del estrés oxidativo, ${ }^{24}$ como ha sido evidenciado en células epiteliales en pulmón y en fibroblastos gingivales humanos. ${ }^{18,20}$ Adicionalmente, se ha reportado ${ }^{3}$ que el cigarrillo convencional o los extractos generados a partir de su consumo son potentes inhibidores de la movilidad espermática reduciendo el potencial de membrana mitocondrial, el cual juega un papel importante en la capacidad fecundante del espermatozoide. Un estudio in vitro $^{26}$ ha revelado que los fumadores tienen un alto número 

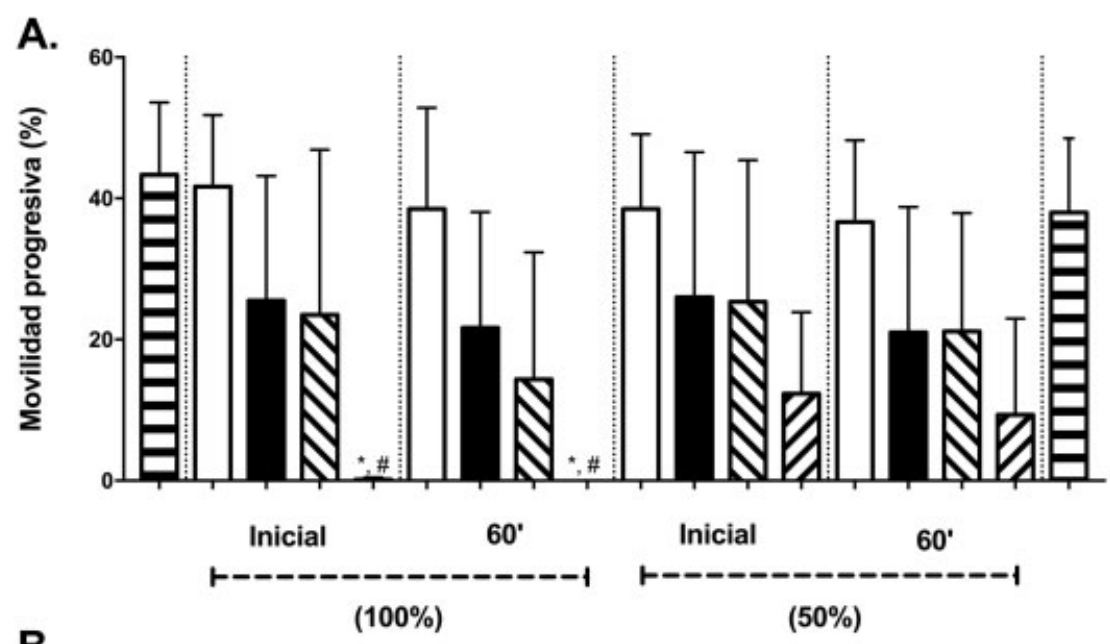

B.

(100\%)

$(50 \%)$

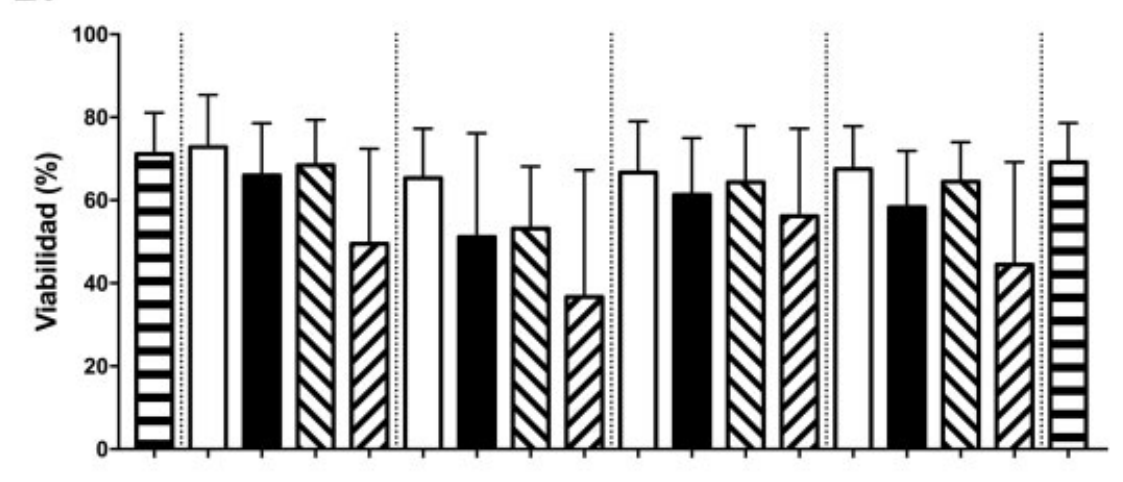

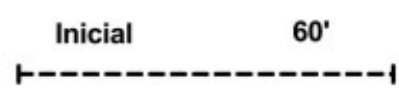

$(100 \%)$

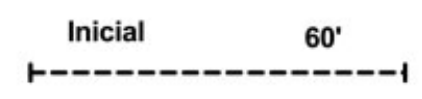

$(50 \%)$

Tiempo

Fig. 4 Efecto de los extractos del cigarrillo electrónico con ambas esencias y cigarrillo convencional sobre (A) la movilidad (\%) y (B) la viabilidad (\%) espermática. * Comparación con respecto a la muestra inicial. " Comparación con respecto a la muestra de aire del mismo tiempo. ${ }^{*} p<0.05$, ${ }^{\#} p<0.05$.

de espermatozoides con anormalidades cromosomales y mayor porcentaje de células en procesos apoptóticos.

Algunos estudios plantean que el cigarrillo electrónico es menos citotóxico que el cigarrillo convencional, como Farsalinos y col., ${ }^{19}$ los cuales compararon el potencial citotóxico del extracto del humo del cigarrillo convencional con el extracto del vapor del cigarrillo electrónico en cultivo de células del miocardio, células de un órgano con conocidas disfunciones causadas a partir del uso del cigarrillo convencional y reportan que no son afectadas por el vapor del E-Cig. Sin embargo, Hyun-Woon Lee y col., ${ }^{27}$ plantean que el vapor del E-Cig produce daños en el ADN y reduce la capacidad de reparación del pulmón, corazón y vejiga de ratones e incluso afecta in vitro las células humanas de pulmón y vejiga.

\section{Conclusiones}

Los resultados del presente trabajo, permiten postular que tanto el vapor del E-Cig como el extracto generado a partir de su uso, afectan la movilidad y la viabilidad de los espermatozoides y que, aunque esos efectos negativos sean menores que los ocasionados por el cigarrillo convencional, seguramente tendrán una repercusión negativa sobre la calidad celular y repercutirán negativamente en la salud de los consumidores.

\section{Responsabilidades Éticas}

Protección de personas y animales: Los autores declaran que para esta investigación no se han realizado experimentos en seres humanos ni en animales.

Confidencialidad de los datos: Los autores declaran que en este artículo no aparecen datos de pacientes.

Derecho a la privacidad y consentimiento informado: Los autores han obtenido el consentimiento informado de los pacientes $y / 0$ sujetos referidos en el artículo. Este documento obra en poder del autor de correspondencia.

Conflicto de Intereses

Los autores declaran no tener ningún conflicto de intereses. 


\section{Referencias}

1 Organización Mundial de la Salud. INFORME OMS SOBRE LAEPIDEMIA MUNDIAL DE TABAQUISMO, 2008. Organización Mundial de la Salud; 2008. http://apps.who.int/iris/bitstream/handle/10665/43897/97892 43596280_spa.pdf;jsessionid=60D49395D7D65B169240B71D26B83 809?sequence=1 (Visto en: 17 abril 2018).

2 Centros para el Control y la Prevención de Enfermedades (CDC). Informe de la Cirujana General de los Estados Unidos: El humo del tabaco causa enfermedades: cómo le afecta a usted: Centros para el Control y la Prevención de Enfermedades (CDC); 2010. https:// www.cdc.gov/tobacco/data_statistics/sgr/2010/consumer_book let/spanish/pdfs/consumer_sp.pdf (Visto en: 22 abril 2018).

3 Calogero A, Polosa R, Perdichizzi A, et al. Cigarette smoke extract immobilizes human spermatozoa and induces sperm apoptosis. Reprod Biomed Online 2009;19(04):564-571

4 Mostafa T. Cigarette smoking and male infertility. J Adv Res 2009; 1(03):179-186

5 Kulikauskas V, Blaustein D, Ablin RJ. Cigarette smoking and its possible effects on sperm. Fertil Steril 1985;44(04):526-528

6 Vine MF, Tse CK, Hu P, Truong KY. Cigarette smoking and semen quality. Fertil Steril 1996;65(04):835-842

7 Sharma R, Harlev A, Agarwal A, Esteves SC. Cigarette Smoking and Semen Quality: A New Meta-analysis Examining the Effect of the 2010 World Health Organization Laboratory Methods for the Examination of Human Semen. Eur Urol 2016;70(04):635-645

8 Jenkins TG, Aston KI, Meyer TD, et al. Decreased fecundity and sperm DNA methylation patterns. Fertil Steril 2016;105(01): 51-7.e1, 3

9 Lerner CA, Sundar IK, Yao H, et al. Vapors produced by electronic cigarettes and e-juices with flavorings induce toxicity, oxidative stress, and inflammatory response in lung epithelial cells and in mouse lung. PLoS One 2015;10(02):e0116732

10 Sancilio S, Gallorini M, Cataldi A, di Giacomo V. Cytotoxicity and apoptosis induction by e-cigarette fluids in human gingival fibroblasts. Clin Oral Investig 2016;20(03):477-483

11 Pauly J, Li Q, Barry MB. Tobacco-free electronic cigarettes and cigars deliver nicotine and generate concern. Tob Control 2007;16 (05):357-360

12 Truman P, Glover M, Fraser T. An Online Survey of New Zealand Vapers. Int J Environ Res Public Health 2018;15(02):1-14

13 Rezk BM, Sikka SC, Hellstrom WJG. Effects of electronic cigarette on men's sexual and reproductive health. Academic Press (Ed). Bioenvironmental Issues Affecting Men's Reproductive and Sexual Health, 1 ed.; 2017:483-491

14 Polosa R, Caponnetto P, Morjaria JB, Papale G, Campagna D, Russo C. Effect of an electronic nicotine delivery device (e-Cigarette) on smoking reduction and cessation: a prospective 6-month pilot study. BMC Public Health 2011;11(786):786

15 Besaratinia A, Tommasi S. Electronic cigarettes: the road ahead. Prev Med 2014;66:65-67

16 FEMA. The Flavor Industry: From Ancient Times to Today. https:// www.femaflavor.org/the-flavor-industry-from-ancient-times-to -today(Visto en: 10 mayo2018)

17 Goniewicz ML, Knysak J, Gawron M, et al. Levels of selected carcinogens and toxicants in vapour from electronic cigarettes. Tob Control 2014;23(02):133-139

18 Cervellati F, Muresan XM, Sticozzi C, et al. Comparative effects between electronic and cigarette smoke in human keratinocytes and epithelial lung cells. Toxicol In Vitro 2014;28(05):999-1005

19 Farsalinos KE, Romagna G, Allifranchini E, et al. Comparison of the cytotoxic potential of cigarette smoke and electronic cigarette vapour extract on cultured myocardial cells. Int J Environ Res Public Health 2013;10(10):5146-5162

20 Romagna G, Allifranchini E, Bocchietto E, Todeschi S, Esposito M, Farsalinos KE. Cytotoxicity evaluation of electronic cigarette vapor extract on cultured mammalian fibroblasts (ClearStream-LIFE): comparison with tobacco cigarette smoke extract. Inhal Toxicol 2013;25(06):354-361

21 Azzopardi D, Patel K, Jaunky T, et al. Electronic cigarette aerosol induces significantly less cytotoxicity than tobacco smoke. Toxicol Mech Methods 2016;26(06):477-491

22 Organización Mundial de la Salud. WHO laboratory manual for the examination and processing of human semen. behalf of the World Health Organization by Medica Panamericana. 2010: 21-30 http:// www.who.int/reproductivehealth/publications/infertility/978924 1547789/en/ (Visto en: 2 febrero 2018)

23 El Golli N, Rahali D, Jrad-Lamine A, et al. Impact of electroniccigarette refill liquid on rat testis. Toxicol Mech Methods 2016;26 (06):427-434

24 Mosbah R, Yousef MI, Mantovani A. Nicotine-induced reproductive toxicity, oxidative damage, histological changes and haematotoxicity in male rats: the protective effects of green tea extract. Exp Toxicol Pathol 2015;67(03):253-259

25 Oyeyipo IP, Raji Y, Bolarinwa AF. Antioxidant profile changes in reproductive tissues of rats treated with nicotine. J Hum Reprod Sci 2014;7(01):41-46

26 Gandini L, Lombardo F, Lenzi A, et al. The in-vitro effects of nicotine and cotinine on sperm motility. Hum Reprod 1997;12(04):727-733

27 Lee HW, Park SH, Weng MW, et al. E-cigarette smoke damages DNA and reduces repair activity in mouse lung, heart, and bladder as well as in human lung and bladder cells. Proc Natl Acad Sci U S A 2018;115(07):E1560-E1569 